\title{
Improved laparoscopic nephron-sparing surgery for renal cell carcinoma based on the precise anatomy of the nephron
}

\author{
GANG GUO, WEI CAI and XU ZHANG \\ Department of Urology, General Hospital of the People's Liberation Army, Beijing 100853, P.R. China
}

Received March 11, 2015; Accepted January 7, 2016

DOI: $10.3892 / 01.2016 .5159$

\begin{abstract}
The aim of the present study was to investigate a method of laparoscopic nephron-sparing surgery (LNSS) for renal cell carcinoma (RCC) based on the precise anatomy of the nephron, and to decrease the incidence of hemorrhage and urinary leakage. Between January 2012 and December 2013, 31 patients who presented to the General Hospital of the People's Liberation Army (Beijing, China) were treated for RCC. The mean tumor size was $3.4 \pm 0.7 \mathrm{~cm}$ in diameter (range, 1.2-6.0 cm). During surgery, the renal artery was blocked, and subsequently, an incision in the renal capsule and renal cortex was performed, at 3-5 $\mathrm{mm}$ from the tumor edge. Subsequent to the incision of the renal parenchyma, scissors with blunt and sharp edge were used to separate the base of the tumor from the normal renal medulla, in the direction of the ray medullary in the renal pyramids. The basal blood vessels were incised following the hemostasis of the region using bipolar coagulation. The minor renal calyces were stripped carefully and the wound was closed with an absorbable sutures. The arterial occlusion time, duration of surgery, intraoperative bleeding volume, post-operative drainage volume, pathological results and complications were recorded. The surgery was successful for all patients. The estimated average intraoperative bleeding volume was $55.7 \mathrm{ml}$, the average surgical duration was $95.5 \mathrm{~min}$, the average arterial occlusion time was $21.2 \mathrm{~min}$, the average post-operative drainage volume was $92.3 \mathrm{ml}$ and the average post-operative length of hospital stay was 6.1 days. No hemorrhage or urinary leakage was observed in the patients following the surgery. LNSS for RCC based on the precise anatomy of the nephron was concluded to be effective and feasible. The surgery is useful for the complete removal of tumors and guarantees a negative margin, which may also decrease the incidence of hemorrhage and urinary leakage following surgery.
\end{abstract}

Correspondence to: Dr Xu Zhang, Department of Urology, General Hospital of the People's Liberation Army, 28 Fuxing Road, Beijing 100853, P.R. China

E-mail: xu_zhang0209@163.com

Key words: renal tumor, laparoscopy, partial nephrectomy

\section{Introduction}

Renal cell carcinoma (RCC) is the most frequently occurring type of kidney cancer in adults. RCC accounts for $3 \%$ of adult malignancies and $90-95 \%$ of neoplasms that arise from the kidney (1). RCC may remain clinically occult for the majority of its course, with only $10 \%$ of patients presenting to hospital with flank pain, hematuria and a flank mass, the classic triad of symptoms (2). The only treatment known to be effective against localized RCC is surgical resection, which may also be used for palliation in metastatic disease. Nephron-sparing surgery (NSS) refers to the complete resection of the tumor and the simultaneous effective retainment of renal tissue in order to maximize renal function (3). With the development of iconography and the popularity of physical examinations, the detection rate of incidental renal tumors and small renal carcinomas without symptoms has increased markedly, and increasing numbers of patients with renal tumors choose to receive NSS. Laparoscopy technology has widespread applications in urinary surgery. Laparoscopic NSS (LNSS) has become more common in the treatment of RCC and has the advantages of little trauma, quick recovery and a similar effect to open surgery (4). The core technologies of LNSS include controlling the warm ischemia time, guaranteeing a negative margin and avoiding the occurrence of secondary bleeding and urine leakage (5). Based on the constantly updated knowledge regarding renal anatomy and the anatomical structure of the nephron, certain improvements were made to the LNSS technique, which was then used to treat 31 patients with RCC in the present study. The improved LNSS achieved good clinical results.

\section{Materials and methods}

Clinical data. A total of 56 patients, including 35 males and 21 females, who presented with RCC to the General Hospital of the People's Liberation Army (Beijing, China) were treated between January 2012 and November 2014. The mean age of the patients was $54.1 \pm 13.2$ years, with a range of $28-68$ years. All patients were indicated to possess space-occupying lesions of the kidney during the physical examinations, and were subsequently hospitalized without clinical symptoms. Prior to surgery, an ultrasound (Pro Focus 2202 Ultrasound Scanner, BK Ultrasound, Herlev, Denmark), computed tomography angiography (CTA) scan (SOMATOM ${ }^{\circledR}$ Definition AS+, Siemens AG, Munich, Germany) and magnetic resonance 
Table I. Clinical comparison of patient features between the groups.

\begin{tabular}{|c|c|c|c|}
\hline Feature & Improved group $(\mathrm{n}=56)$ & Control group $(n=36)$ & P-value \\
\hline Age, years ${ }^{\mathrm{a}}$ & $54.1 \pm 13.2$ & $52.3 \pm 10.7$ & 0.46 \\
\hline Male gender, n (\%) & $35(62.5)$ & $23(63.9)$ & 0.80 \\
\hline $\mathrm{BMI}^{\mathrm{a}}$ & $22.3 \pm 1.2$ & $23.3 \pm 1.6$ & 0.93 \\
\hline Tumor diameter, $\mathrm{cm}^{\mathrm{a}}$ & $3.1 \pm 0.7$ & $3.4 \pm 1.1$ & 0.56 \\
\hline \multicolumn{4}{|l|}{ Affected side, n (\%) } \\
\hline Left & $25(44.6)$ & $15(41.7)$ & 0.67 \\
\hline Right & $31(55.4)$ & $21(58.3)$ & 0.38 \\
\hline \multicolumn{4}{|l|}{ Location } \\
\hline Upper pole & $20(35.7)$ & $14(38.9)$ & 0.57 \\
\hline Middle & $22(39.3)$ & $12(33.3)$ & 0.34 \\
\hline Inferior pole & $14(25.0)$ & $10(27.8)$ & 0.76 \\
\hline R.E.N.A.L. ${ }^{a}$ & $6.9 \pm 1.1$ & $6.6 \pm 0.8$ & 0.75 \\
\hline GFR prior to surgery, $\mathrm{ml} / \mathrm{min}^{\mathrm{a}}$ & $45.6 \pm 6.4$ & $47.6 \pm 7.4$ & 0.89 \\
\hline
\end{tabular}

${ }^{a}$ Data are expressed as mean \pm standard deviation. BMI, body mass index; GFR, glomerular filtration rate; R.E.N.A.L., R.E.N.A.L. nephrometry score, as determined by radius, exophytic/endophytic properties, nearness of tumor to the collecting system or sinus, anterior/posterior and location to relative polar lines.

imaging (MRI; MAGNETOM Avanto ${ }^{\mathrm{TM}}$ 3.0T, Siemens AG) examination were performed, in order to evaluate the renal vessels and location of the tumor. The average tumor size was $3.1 \pm 0.7 \mathrm{~cm}$ in diameter, and ranged from $1.2-4.0 \mathrm{~cm}$ (Table I). Pre-operative tumor-node-metastasis (TNM) staging (6) showed that 53 cases were T1a and 3 were T1b, including one with an anatomically solitary kidney and two with chronic renal insufficiencies. The pre-operative diagnoses were all considered to be renal tumors. The complicating diseases included 4 patients with diabetes, 2 patients with chronic renal insufficiency and 5 patients with hypertension. Routine examinations prior to the surgery revealed no surgical contraindications. In addition, a control group was studied. The control group consisted of 36 patients that received traditional partial nephrectomy surgery, which involves a sharp incision of the tumor with scissors, $5 \mathrm{~mm}$ from the tumor margin.

Surgical method. All patients received LNSS under general anesthetic. Following successful anesthesia, the patients were placed in the lateral decubitus position. Trocars that were 5, 10 and $12 \mathrm{~mm}$ in diameter were placed $1 \mathrm{~cm}$ above the iliac crest in the midaxillary line, and under the 12th costal margin at the anterior and posterior axillary lines, respectively. Next, laparoscopic and surgical apparatus were implanted. The $\mathrm{CO}_{2}$ pressure of the pneumoperitoneum was maintained at $10.5 \mathrm{~mm} \mathrm{Hg} \mathrm{H}_{2} \mathrm{O}$. Following the establishment of peritoneal clearance, the perirenal fascia was opened and the renal tumor was fully exposed. The renal arteries were dissociated and reattached in front of the psoas major muscle, using a Delacroix-Chevalier Gregory Bulldog clamp (115 mm; Landanger, Paris, France) to control the renal artery, and the timer was started.

Subsequent to blocking the renal artery, the renal capsule and renal cortex were incised using scissors with a ring-like

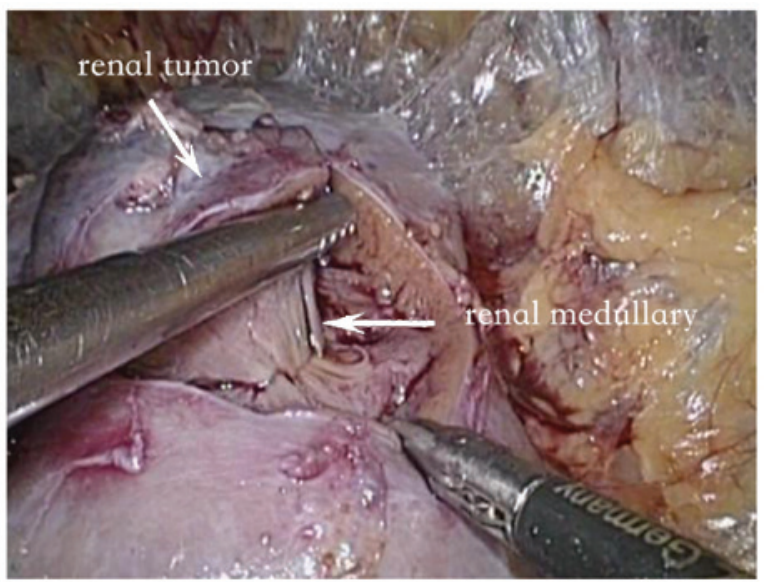

Figure 1. Separation of the tumor and reserved medullary. Following the incision of the renal parenchyma, scissors with blunt and sharp edge were used to separate the base of the tumor from the normal renal medulla, in the direction of the ray medullary in the renal pyramids.

shape (34310-MA-D; Karl Storz, Tuttlingen, Germany) at 3-5 $\mathrm{mm}$ from the tumor edge. Following the incision of the renal parenchyma, the tumor was separated from the reserved medullary and medullary ray to the depth of the basement membrane (Fig. 1). The basal blood vessels were incised, subsequent to the hemostasis of the region using bipolar coagulation (Fig. 2). The minor renal calyces were carefully peeled from the structure to reveal the clear anatomical structure of the wound (Fig. 3). The basal damaged blood vessels and collecting systems were continuously sealed with 2-0 absorbable sutures and the surgical wounds were continuously sealed with 1-0 absorbable sutures, using a Weck Hem-o-lok clip (Teleflex, Morrisville, NC, USA) to maintain the tension of the sutures. The Bulldog clamps were released, a lack of bleeding from the wound was confirmed and 
Table II. Comparison of clinical data subsequent to surgery.

\begin{tabular}{|c|c|c|c|}
\hline Feature & Improved group $(n=56)$ & Control group $(n=36)$ & P-value \\
\hline Arterial occlusion time, min & $21.2 \pm 7.2$ & $20.1 \pm 5.7$ & 0.96 \\
\hline Surgical duration, min & $95.5 \pm 27.1$ & $90.5 \pm 21.3$ & 0.47 \\
\hline Intraoperative bleeding, $\mathrm{ml}$ & $55.7 \pm 18.9$ & $63.5 \pm 20.1$ & 0.53 \\
\hline Post-operative drainage, $\mathrm{ml}$ & $92.3 \pm 28.9$ & $112.3 \pm 34.5$ & 0.16 \\
\hline Length of hospital stay, days & $6.1 \pm 0.6$ & $6.9 \pm 0.9$ & \\
\hline \multicolumn{4}{|l|}{ Pathological types, n (\%) } \\
\hline $\mathrm{CCE}$ & $51(91.1)$ & $31(86.1)$ & 0.48 \\
\hline Other & $5(8.9)$ & $5(13.9)$ & 0.11 \\
\hline Negative margin, \% & 100.0 & 88.9 & $<0.01$ \\
\hline \multicolumn{4}{|l|}{ Complications, $\mathrm{n}$} \\
\hline Bleeding or urine leakage & 0 & 5 & $<0.01$ \\
\hline Other & 5 & 5 & 0.13 \\
\hline $\mathrm{GFR}, \mathrm{ml} / \mathrm{min}$ & $14.32 \pm 2.12$ & $16.17 \pm 2.34$ & 0.34 \\
\hline 1 -year recurrence rate, $\%$ & 0.0 & 2.8 & 0.76 \\
\hline
\end{tabular}

CCE, clear-cell carcinoma; GFR, glomerular filtration rate.

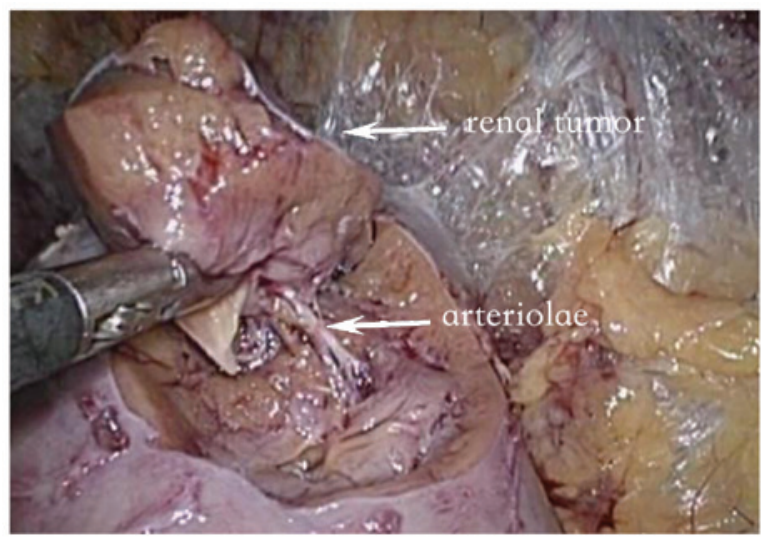

Figure 2. Clear straight arterioles at the base of the kidney.

the blood supply of the kidney returned to normal. A drain was left following the surgery. The arterial occlusion time, surgical duration, intraoperative bleeding volume, post-operative drainage volume, pathological results, complications and post-operative follow-up results were recorded.

All the tumor specimens collected from the surgery were embedded in paraffin, sectioned and stained with hematoxylin and eosin (HE). The pathological subtypes of renal cell carcinoma were observed and analyzed by two professional pathologists, using HE staining.

\section{Results}

Surgery. All 31 patients were operated on successfully and no cases were converted to open surgery. The surgical duration was $80-120 \mathrm{~min}$ (mean, 95.5 $\pm 27.1 \mathrm{~min}$ ) and the arterial occlusion times were 15-30 $\mathrm{min}$ (mean, 21.2 $\pm 7.2 \mathrm{~min}$ ). The average intraoperative bleeding volume was $55.7 \pm 18.9 \mathrm{ml}$, with a

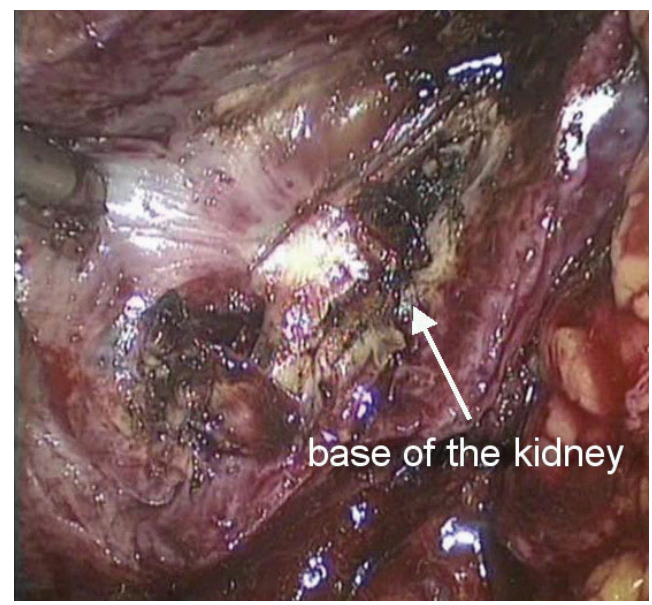

Figure 3. Wound with clear anatomical structure following the stripping of the minor renal calyces.

range of 30-150 $\mathrm{ml}$, while the average post-operative drainage volume was $92.3 \pm 28.9 \mathrm{ml}$, with a range of $50-250 \mathrm{ml}$. The average post-operative length of hospital stay was $6.1 \pm 0.6$ days, ranging from 5-7 days. No hemorrhage or urinary leakage was observed in any patients following the surgery (Table II).

Post-surgery. The post-operative pathological results indicated that 27 cases were diagnosed with suprarenal epithelioma, 2 with chromophobe cell renal carcinoma, 1 with oxyphilic adenoma and 1 with a juxtaglomerular cell tumor. The post-operative TNM staging revealed that 28 patients possessed Tla stage tumors and 3 patients possessed stage T1b tumors. The Fuhrman classification (7) was used to classify 15 patients with level 1, 8 cases with level 1-2 and 8 cases with level 2 RCC. All tumor specimens that were removed were wedge-shaped. The tumors were well circumscribed with negative margins. 
Following discharge from the hospital, all patients were followed up for 8-28 months (mean, 18.5 \pm 1.6 months) and no signs of local recurrence or distant metastases were identified by renal ultrasound or computed tomography examinations.

\section{Discussion}

Radical nephrectomies may lead to renal decompensation. Therefore, for patients who have kidney ailments, which may endanger renal function, or renal tumors at the T1a clinical stage, LNSS is the recommended surgery (8). The core technologies of LNSS include controlling the warm ischemia time, guaranteeing a negative margin and avoiding the occurrence of secondary bleeding and urine leakage (5). Continuous research and improvements have been developed for these surgical aspects, including suture techniques, renal hypothermia protection technology, hemostatic materials and ureteral stents (9-12). These beneficial improvements ensure that LNSS is continuously improved and developed, and the clinical applications of LNSS are expanding.

The direction of the laparoscopic operative channel limits the surgery, and certain disadvantages remain unavoidable, including the narrow operative space. Occasionally, the cutting position is not visible and judging the base of the tumor is challenging, which may result in cutting more renal tissue or cutting into the tumor. Therefore, certain cases require surgeons with increased surgical experience and no standard exists to aid the judgment of the anatomical base of the tumor (13). At present, incisions with an ultrasound knife or sharp cuts with scissors are in common use in LNSS, which may easily damage the tumor capsule. In order to prevent the recurrence of a tumor following the partial nephrectomy of renal tumors, tumors have always been cut along the normal tissue to guarantee a negative margin (14). Through the investigation of multiple centers, a study conducted by Breda et al indicated that 21/855 (2.46\%) patients who received LNSS had a positive margin (15). Urinary leakage is the major complication following LNSS, and dealing with the collecting system may extend the surgery and the warm ischemia time; therefore, doctors are required to be skilled in the associated surgical techniques. Effectively closing the collecting system during surgery may greatly decrease the possibility of urinary leakage post-operatively (16).

The renal parenchyma is composed of the renal cortex and the renal medulla, in a 1:2 ratio. Renal tumors grow in the renal parenchyma under the renal capsule and show inflated growth. Renal tumors continuously squeeze the surrounding renal parenchyma to form a pseudocapsule, and traditionally, the excision of renal tumors is always performed along the pseudocapsule. The renal capsule is composed mainly of fibrous tissue, and the renal cortex consists of a number of renal corpuscles, which are dense (17). The renal medulla includes numerous straight renal tubules that are arranged radially to form the medullary ray and the renal pyramids. Renal pyramids and the corresponding renal cortex are composed of renal lobules. The majority of the straight arterioles of the renal medulla are parallel to the long axis of the renal pyramids (18). The amplification effects of the laparoscope allow the easy identification of these precise anatomical structures.

In the present study, subsequent to cutting the renal capsule and renal parenchyma, the separation of the tumor from the reserved medullary and medullary ray was performed to the depth of the basement membrane, which is a simple procedure. The tumor was isolated with visible supplying blood vessels in 27 cases. The blood vessels were cut following the hemostasis of the region using bipolar coagulation, the wounds of which were simple to suture and possessed a low possibility of bleeding. In addition, for the central type of renal tumor, or 'H' in the R.E.N.A.L. nephrometry scoring system, the tumors are recommended to be stripped along the renal medulla to the renal sinus, subsequent to opening the renal cortex, in order to avoid the accidental injury of the renal artery and vein (19). The tumors of 3 patients with the central type of renal tumor in the present study were removed completely, and the renal artery and vein were not damaged; therefore, this method may also be applied to the treatment of central-type renal tumors. Urine collecting systems include the minor renal calyces, renal calyces, renal pelvis and ureteropelvic junction. The renal parenchyma encompasses the minor renal calyces, renal calyces and the majority of the renal pelvis. A renal calyx is composed of 2 or 3 minor renal calyces. Carefully stripping the base of the tumor may prevent the injury of the minor renal calyces (20). Fine processing of the blood vessels, renal medulla and minor renal calyces may also decrease the flow of blood from the surface of the wound, which reveals the structure clearly and provides a good view for the suturing. Therefore, the arterial occlusion times of the surgery reported in the present study were similar compared with previous reports in the literature.

Our preliminary experience of using the present method indicated the following features: i) The integrity of the tumor may be guaranteed during treatment for deep and basal tumors. Since the surgery was performed along the separated medullary space, the tumor was protected and the excision of excessive renal tissue was avoided. ii) Blood vessels that supply the tumor may be isolated and treated separately in order to decrease the risk of secondary bleeding. iii) The minor renal calyces may be isolated and treated separately to decrease the possibility of urinary leakage. Overall, the improved LNSS for RCC, based on the precise anatomy of the nephron, allows the excision of renal tumors according to the renal lobules and renal pyramids. The surgery ensures the complete resection of the tumor without the removal of excessive renal tissue and has the effect of precisely excising the tumor and the accumulating nephron. The LNSS method is simple, easy to master, and has the advantages of a low positive margin rate and fewer post-operative complications. In addition, the warm ischemia time was not increased during surgery, which may protect renal function to a greater extent, and the method is suitable for promoting for clinical use. The short-term treatment effects of the surgery are satisfactory, but the long-term effects require additional large prospective, randomized controlled studies to confirm the success of LNSS.

\section{References}

1. American Cancer Society: Cancer Facts \& Figures 2014 http://www.cancer.org/acs/groups/content/@research/documents/web content/acspc-042151.pdf. Accessed June 1, 2014.

2. Simon JW, Marshall FF: Kidney and ureter. In: Clinical Oncology. Abeloff MD, Armitage J, Niederhuber J, Kastan M and McKenna W (eds). 2nd edition. Churchill Livingstone, New York, NY, pp1784-1799, 2000.

3. Margreiter M and Marberger M: Current status of open partial nephrectomy. Curr Opin Urol 20: 361-364, 2010. 
4. Schwaibold HE and Stolzenburg JU: Laparoscopic partial nephrectomy. Arch Ital Urol Androl 81: 72-75, 2009.

5. Pietzak EJ and Guzzo TJ: Advancements in laparoscopic partial nephrectomy: Expanding the feasibility of nephron-sparing. Adv Urol 2012: 148952, 2012.

6. Belldegrun A, Tsui KH, deKernion JB and Smith RB: Efficacy of nephron-sparing surgery for renal cell carcinoma: Analysis based on the new 1997 tumor-node-metastasis staging system. J Clin Oncol 17: 2868-2875, 1999.

7. López JI: Comment to «Is a new classification of the Fuhrman grade in clear cell renal cell carcinomas feasible?». Actas Urol Esp 36: 359-360, 2012 (In Spanish).

8. Aron M and Turna B: Laparoscopic partial nephrectomy: Newer trends. Indian J Urol 25: 516-522, 2009.

9. Zorn KC, Gong EM, Orvieto MA, Gofrit ON, Mikhail AA and Shalhav AL: Impact of collecting-system repair during laparoscopic partial nephrectomy. J Endourol 21: 315-320, 2007.

10. Gill IS, Ramani AP, Spaliviero M, Xu M, Finelli A, Kaouk JH and Desai MM: Improved hemostasis during laparoscopic partial nephrectomy using gelatin matrix thrombin sealant. Urology 65 463-466, 2005

11. Nozaki T, Iida H, Morii A, Fujiuchi Y and Fuse H: Selective renal parenchymal clamping in retroperitoneal partial nephrectomy. J Laparoendosc Adv Surg Tech A 22: 168-172, 2012.

12. Wen J, Li HZ, Ji ZG, Shi BB and Yan WG: Evaluation of retroperitoneoscopic partial nephrectomy with in situ hypothermic perfusion. Clin Transl Oncol 14: 382-385, 2012.
13. Kong W, Zhang J, Dong B, Chen Y, Xue W, Liu D and Huang Y: Application of a standardized anatomical classification in a Chinese partial nephrectomy series. Int J Urol 19: 551-558, 2012.

14. Springer C, Hoda MR, Fajkovic H, Pini G, Mohammed N, Fornara Pand Greco F: Laparoscopic vs open partial nephrectomy for T1 renal tumours: Evaluation of long-term oncological and functional outcomes in 340 patients. BJU Int 111: 281-288, 2013.

15. Breda A, Stepanian SV, Liao J, Lam JS, Guazzoni G, Stifelman M, Perry K, Celia A, Breda G, Fornara P, et al: Positive margins in laparoscopic partial nephrectomy in 855 cases: A multi-institutional survey from the United States and Europe. J Urol 178: 47-50, 2007.

16. Wang P, Xia D and Wang S: Multiple factor analysis of urine leaks after retroperitoneal laparoscopic partial nephrectomy. Urol Int 87: 411-415, 2011.

17. Sampaio FJ: Renal anatomy. Endourologic considerations. Urol Clin North Am 27: 585-607, 2000.

18. Preuss HG: Basics of renal anatomy and physiology. Clin Lab Med 13: 1-11, 1993.

19. Okhunov Z, Shapiro EY, Moreira DM, Lipsky MJ, Hillelsohn J, Badani K, Landman J and Kavoussi LR: R.E.N.A.L. nephrometry score accurately predicts complications following laparoscopic renal cryoablation. J Urol 188: 1796-1800, 2012.

20. Stroup SP, Palazzi K, Kopp RP, Mehrazin R, Santomauro M, Cohen SA, Patterson AL, L'Esperance JO and Derweesh IH: RENAL nephrometry score is associated with operative approach for partial nephrectomy and urine leak. Urology 80: 151-156, 2012. 